UDC $681.5(045)$

${ }^{1}$ V. V. Chikovani,

${ }^{2}$ H. V. Tsiruk

\title{
BIAS COMPENSATION IN DIFFERENTIAL CORIOLIS VIBRATORY GYRO
}

Institute of Air Navigation, National Aviation University, Kyiv, Ukraine E-mails: ${ }^{1}$ valeriy chikovani@rambler.ru, ${ }^{2}$ annylee@ukr.net

\begin{abstract}
This work presets one of the ways to compensate for the bias in differential mode of Coriolis vibratory gyro operation. To compensate for the bias it is necessary to know both channels scale factors, $S F_{x}$ and $S F_{y}$ of differential Coriolis vibratory gyro, which, as a rule, are known by the results of calibration procedure and elastic wave angle $\theta$ relative to one of the two drive axes which can be established in advance. This paper presents the different variants of bias compensation algorithms which can be applied to compensate for initial gyro bias and bias drift in motion. Experimental results on differential Coriolis vibratory gyro key parameters temperature influence are also presented.
\end{abstract}

Keywords: differential Coriolis vibratory gyro; bias; standing wave angle; phases difference.

\section{INTRODUCTION}

The differential mode of operation, which is analyzed in this paper, can be implemented in the ring-like Coriolis vibratory gyro (CVG) resonator by keeping a standing wave between the electrodes by applying two stable amplitude signals on $X$ and $Y$ drive axes. In this case two magnitudes of angular rates with opposite signs can be read-out from $X$ and $Y$ sense axes [1]. The resulting angular rate can be obtained by signals subtraction of two angular rate channels and at proper alignment of standing wave cross damping bias component is compensated. Moreover, the sum of these two signals gives information about the bias drift components without angular rate that can be used to estimate certain bias components for on-line compensation [2].

\section{PROBLEM STATEMENT}

Differential CVG gives many possibilities to estimate and/or to compensate for bias drift. Some of bias compensation algorithms are derived and discussed in this paper. Experimental results on differential CVG key parameters temperature influence are also presented.

\section{DIFFERENTIAL CVG MEASUREMENT EQUATIONS}

Suppose that under applying control signals $f_{x}$ and $f_{y}$ to the $X$ and $Y$ electrodes the standing wave is aligned between them, as it is shown by the dashed line in Fig. 1. The dynamic equations of the standing wave in this case can be written down as follows [3]:

$$
\begin{aligned}
& \ddot{x}+d_{x x} \dot{x}+k_{x x} x+k_{x y} y=\left(2 k \Omega-d_{x y}\right) \dot{y}+f_{x}, \\
& \ddot{y}+d_{y y} \dot{y}+k_{x y} x+k_{y y} y=\left(-2 k \Omega-d_{x y}\right) \dot{x}+f_{y},
\end{aligned}
$$

where $k$ is the Brian coefficient, approximately equal to $0.4 ; d_{x x}=2 / \tau+h \cos 2 \theta_{\tau}$ is $X$ axis damping coefficient $2 / \tau=1 / \tau_{1}+1 / \tau_{2}, h=1 / \tau_{1}-1 / \tau_{2} ; \tau_{1}$ is the minimum resonator damping time; $\tau_{2}$ is the maximum resonator damping time; $d_{x y}=h \sin 2 \theta_{\tau}$ is the damping cross-coupling; $d_{y x}=d_{x y}$; $k_{x x}=\omega_{1}^{2}-\omega \Delta \omega \cos 2 \theta_{\omega}$ is the resonator rigidity coefficient along $X$ axis normalized by the mass, $\omega \Delta \omega=\left(\omega_{1}^{2}-\omega_{2}^{2}\right) / 2$, where $\omega_{1}$ and $\omega_{2}$ are the maximum and minimum resonant frequencies; $k_{x y}=-\omega \Delta \omega \sin 2 \theta_{\omega}$ is the rigidity cross-coupling; $k_{x y}$ $=k_{y x} ; d_{y y}=2 / \tau-h \cos 2 \theta_{\tau}$ is $Y$ axis damping coefficient; $k_{y y}=\omega_{2}^{2}++\omega \Delta \omega \cos 2 \theta_{\omega}$ is the resonator rigidity coefficient along $Y$ axis normalized by the mass; $f_{x}, f_{y}$ are normalized by the mass control signals; $\theta_{\omega}$ is an angle between minimum frequency axis and standing wave (antinode) axis; $\theta_{\tau}$ is an angle between the minimum damping axis and the standing wave (antinode) axis.

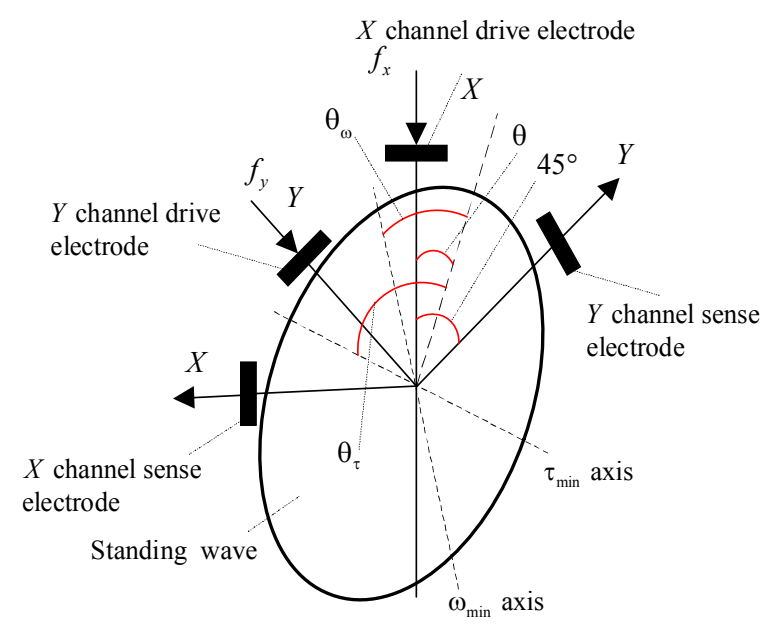

Fig. 1. Standing wave in ring-like differential $\mathrm{CVG}$ resonator 
Control signals $f_{x}$ and $f_{y}$ should be applied on $X$ and $Y$ axes to make the rigidity equal $k_{x x}=k_{y y}$. It means that $\omega_{1}=\omega_{2}=\omega_{r}$.

Let's find stationary solution of equations (1) in view of the following expressions

$$
x=r \cos 2 \theta \sin \left(\omega_{r} t\right), y=r \cos 2 \theta \sin \left(\omega_{r} t+\varphi\right),
$$

where $r$ is oscillation amplitude; $\theta$ is vibration angle relative to $X$ axis; $\varphi$ is phase difference between $X$ and $Y$ sense axes signals that should be constant to provide $\omega_{1}=\omega_{2}=\omega_{r}[2]$.

Suppose that $\theta \neq n \pi / 4, n=0,1,2, \ldots$, then for $X$ and $Y$ sense signals $z_{x}$ and $z_{y}$ in voltages after demodulation by reference signals $\sin \omega_{r} t$ and $\cos \omega_{r} t$ and transformations, the following expressions can be obtained [2]

$$
\begin{array}{r}
-2 k \Omega D_{y} \operatorname{tg} 2 \theta \cos \varphi+d_{x y} D_{y} \operatorname{tg} 2 \theta \cos \varphi+D_{x} d_{x x}=z_{x} ; \\
2 k \Omega D_{x} \cot 2 \theta+d_{x y} D_{x} \operatorname{ctg} 2 \theta+D_{y} d_{y y} \cos \varphi=z_{y},
\end{array}
$$

where $D_{x}$ and $D_{y}$ are transformation coefficients of mechanical deformation into the $X$ and $Y$ electrodes voltages.

\section{BIAS COMPENSATION BY SWITCHING CONTROL}

Coefficients at angle rate $\Omega$ variable are scale factors of the $X$ and $Y$ sense channels

$$
\begin{aligned}
& S F_{x}=2 k D_{y} \operatorname{tg} 2 \theta \cos \varphi ; \\
& S F_{y}=2 k D_{x} \cot 2 \theta .
\end{aligned}
$$

Let $\varphi=0$, then (3) can be rewritten as follows

$$
\begin{aligned}
& -S F_{x} \Omega+d_{x y} D_{y} \operatorname{tg} 2 \theta+D_{x} d_{x x}=z_{x}^{0} ; \\
& S F_{y} \Omega+d_{x y} D_{x} \operatorname{ctg} 2 \theta+D_{y} d_{y y}=z_{y}^{0} .
\end{aligned}
$$

When $\varphi=\pi$, then (3) is

$$
\begin{aligned}
& S F_{x} \Omega-d_{x y} D_{y} \operatorname{tg} 2 \theta+D_{x} d_{x x}=z_{x}^{\pi} ; \\
& S F_{y} \Omega+d_{x y} D_{x} \operatorname{ctg} 2 \theta-D_{y} d_{y y}=z_{y}^{\pi} .
\end{aligned}
$$

After summing and subtracting (5) and (6) the following expressions can be obtained

$$
\begin{aligned}
z_{x}^{0}+z_{x}^{\pi} & =2 D_{x} d_{x x} ; z_{y}^{0}-z_{y}^{\pi}=2 D_{y} d_{y y} ; \\
z_{y}^{0}+z_{y}^{\pi} & =2 S F_{y} \Omega+2 d_{x y} D_{x} \cot 2 \theta ; \\
z_{x}^{\pi}-z_{x}^{0} & =2 S F_{y} \Omega-2 d_{x y} D_{y} \tan 2 \theta .
\end{aligned}
$$

Solution of (7) relative to $\Omega$ yields

$$
\begin{aligned}
z_{y}^{0}+z_{y}^{\pi}+z_{x}^{\pi}-z_{x}^{0} & =2\left(S F_{x}+S F_{y}\right) \Omega \\
& +2 d_{x y}\left(D_{x} \cot 2 \theta-D_{y} \tan 2 \theta\right) ;
\end{aligned}
$$

It can be concluded from (8) that if

$$
D_{x} \cot 2 \theta-D_{y} \tan 2 \theta=0 \text {; }
$$

or $\frac{D_{x}}{D_{y}}=\tan ^{2} 2 \theta ; \rightarrow \theta_{*}=\frac{1}{2} \tan ^{-1} \sqrt{\frac{D_{x}}{D_{y}}}$,

Pure angle rate without bias can be obtained:

$$
\begin{gathered}
z_{y}^{0}+z_{y}^{\pi}+z_{x}^{\pi}-z_{x}^{0}=2\left(S F_{x}^{\theta_{*}}+S F_{y}^{\theta_{*}}\right) \Omega ; \\
S F_{x}^{\theta_{*}}=S F_{x}^{\theta} * T R ; S F_{y}^{\theta_{*}}=S F_{y}^{\theta} / T R ; \\
T R=\frac{1+\tan 2 \Delta \theta}{1-\tan 2 \Delta \theta} ; \Delta \theta=\theta-\theta_{*} .
\end{gathered}
$$

It follows from (4) that

$$
\frac{S F_{y}}{S F_{x}}=\frac{D_{x}}{D_{y}} \cot ^{2} 2 \theta
$$

So, at the beginning wave can be set on arbitrary angle $\theta \neq k \pi / 4, k=0,1,2, \ldots$, more convenient value is $\theta=\pi / 8$. In this case $\cot 2 \theta=1$ and

$$
\frac{S F_{y}^{\pi / 8}}{S F_{x}^{\pi / 8}}=\frac{D_{x}}{D_{y}} \rightarrow \theta_{*}=\frac{1}{2} \tan ^{-1} \sqrt{\frac{S F_{y}^{\pi / 8}}{S F_{x}^{\pi / 8}}} .
$$

Differential CVG control system block diagram is presented in Fig. 2.

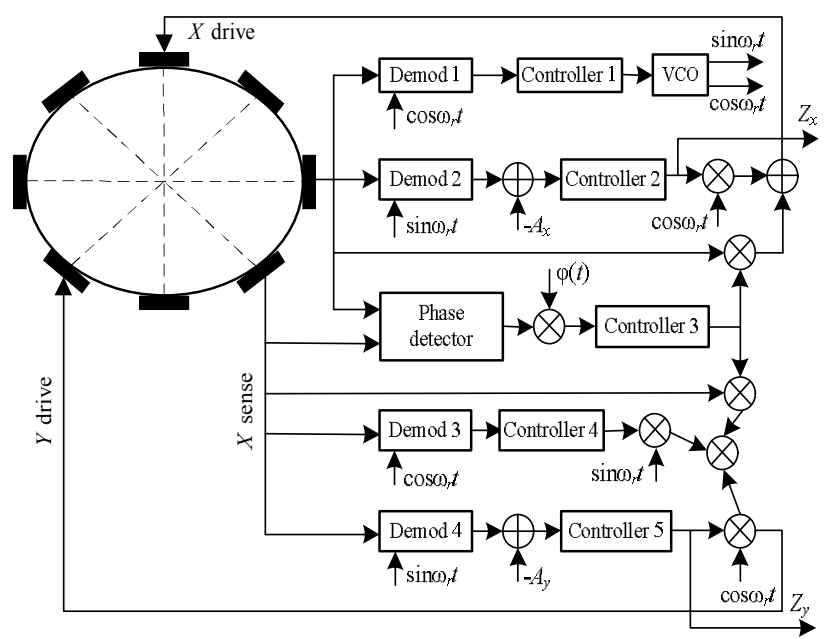

Fig. 2. Differential CVG block diagram

Phase difference $\varphi(t)$ control law to compensate for the differential CVG bias is graphically presented in Fig. 3.

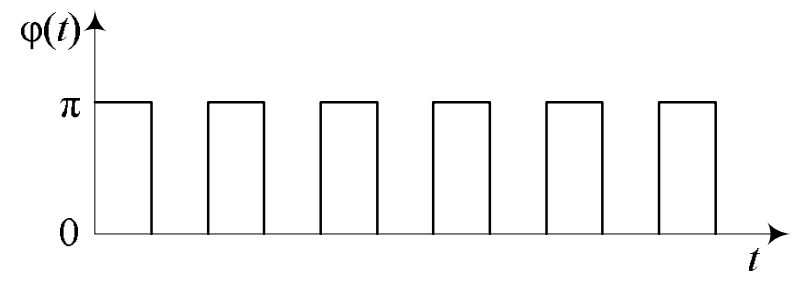

Fig. 3. Phase difference switching control law to compensate for the differential CVG bias 
Because of scale factors $S F_{x}$ and $S F_{y}$ can change versus temperature that results in wave angle $\theta$ change, temperature sensitivity of these parameters should be studied.

Figures 4 and 5 show temperature dependencies of $X$ and $Y$ channels scale factors and total one $S F_{\text {total }}^{\pi / 8}=S F_{x}^{\pi / 8}+S F_{y}^{\pi / 8}$ when wave angle is $\pi / 8$ $(22.5 \mathrm{deg})$. As can be seen from the Fig. $5 S F_{\text {total }}^{\pi / 8}$ temperature sensitivity is $0.016 \% /{ }^{\circ} \mathrm{C}$.
As can be seen from Fig. $4 S F_{x}^{\pi / 8}(T)$ and $S F_{y}^{\pi / 8}(T)$ curves are almost parallel to each other. The latter means that wave angle $\theta$ calculated as a ratio of these two scale factors (see (12)) will be very stable.

Figure 6 shows wave angle $\theta$ calculated by expression (12) for different temperatures. Temperature sensitivity of this angle is sufficiently low and is equal to $0.95 \times 10^{-4} \% /{ }^{\circ} \mathrm{C}$.

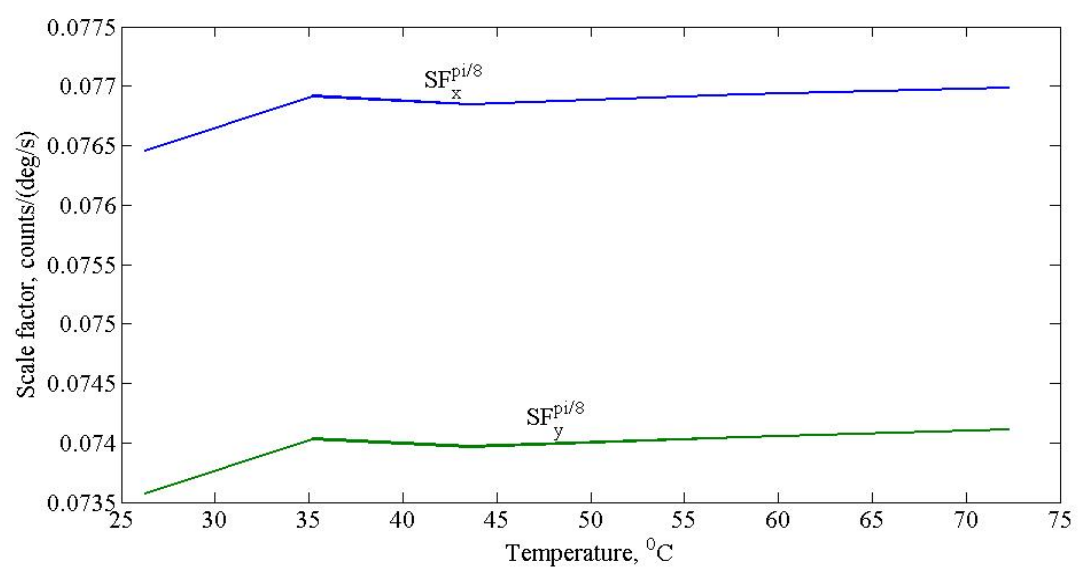

Fig. 4. Scale factors temperature dependence

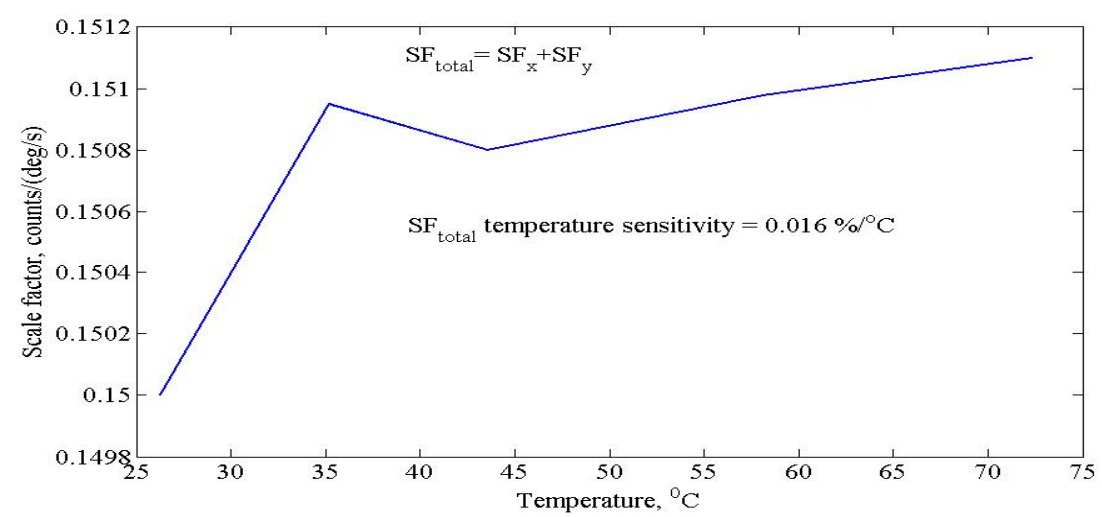

Fig. 5. Total scale factors temperature sensitivity

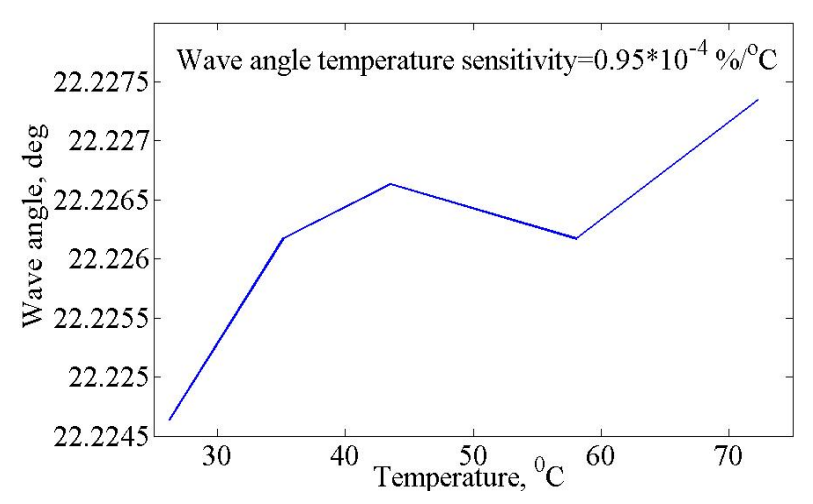

Fig. 6. Wave angle $\theta$ versus temperature

So, first step in differential CVG bias compensation procedure is to align standing wave under angle $\theta=\pi / 8$ and to carry out scale factors $S F_{x}$ and $S F_{y}$ calibration. It can be carried out at room temperature, because their ratio is almost insensitive to temperature change. The second step is to realign the standing wave to the angle $\theta$ in accordance with expression (12). The third step is to send a signal $\varphi(t)$ in accordance with one presented in Fig. 3 to the input of controller 3 (see Fig. 2). Angle rate $\Omega$ should be calculated by he expression (10), where $\theta=\pi / 8$ and as follows from Fig. $6 \Delta \theta=22.5-22.226=0.274 \mathrm{deg}$. (0.00478 rad), $\mathrm{TR}=0.99967$.

\section{BIAS COMPENSATION BY SINE CONTROL}

Switching control law can cause transients which result in measurement errors. To reduce errors in high dynamic measurements sine function control law as depicted in Fig. 7 can be used. 


$$
\varphi(t)=\varphi_{0} \sin \omega_{\varphi} t .
$$

Substitution (13) in (3) yelds
After Bessel function of the first kind expansion of function $\cos \left(\varphi_{0} \sin \omega_{\varphi} t\right)$, the following expressions can be obtained

$$
\begin{aligned}
& \qquad \begin{array}{l}
-2 k \Omega D_{y} \operatorname{tg} 2 \theta \cos \left(\varphi_{0} \sin \omega_{\varphi} t\right)+d_{x y} D_{y} \operatorname{tg} 2 \theta \cos \left(\varphi_{0} \sin \omega_{\varphi} t\right)+D_{x} d_{x x}=z_{x} ; \\
2 k \Omega D_{x} \cot 2 \theta+d_{x y} D_{x} \operatorname{ctg} 2 \theta+D_{y} d_{y y} \cos \left(\varphi_{0} \sin \omega_{\varphi} t\right)=z_{y} .
\end{array} \\
& z_{x}=-S F_{x} J_{0}\left(\varphi_{0}\right) \Omega-2 S F_{x} J_{2}\left(\varphi_{0}\right) \Omega \cos 2 \omega_{\varphi} t+d_{x y} D_{y} \operatorname{tg} 2 \theta J_{0}\left(\varphi_{0}\right)+2 d_{x y} D_{y} \operatorname{tg} 2 \theta J_{2}\left(\varphi_{0}\right) \cos 2 \omega_{\varphi} t+D_{x} d_{x x} ; \\
& z_{y}=S F_{y} \Omega+d_{x y} D_{x} \operatorname{ctg} 2 \theta+D_{y} d_{y y} J_{0}\left(\varphi_{0}\right)+2 D_{y} d_{y y} J_{2}\left(\varphi_{0}\right) \cos 2 \omega_{\varphi} t . \\
& \text { Here only second harmonic of frequency } \omega_{\varphi} \text { is } \quad z_{x}^{B P F D}=-2 S F_{x} J_{2}\left(\varphi_{0}\right) \Omega+2 d_{x y} D_{y} \operatorname{tg} 2 \theta J_{2}\left(\varphi_{0}\right) ; \\
& \text { taking into account. } \\
& \text { Low pass filter (LPF) output signal is }
\end{aligned}
$$

$$
\begin{array}{r}
z_{x}^{L P F}=-S F_{x} J_{0}\left(\varphi_{0}\right) \Omega+d_{x y} D_{y} \operatorname{tg} 2 \theta J_{0}\left(\varphi_{0}\right)+D_{x} d_{x x} ; \\
z_{y}^{L P F}=S F_{y} \Omega+d_{x y} D_{x} \operatorname{ctg} 2 \theta+D_{y} d_{y y} J_{0}\left(\varphi_{0}\right) .
\end{array}
$$

The difference of these two signals yields

$$
\begin{aligned}
& z_{y}^{L P F}-z_{x}^{L P F}=\left(S F_{x} J_{0}\left(\varphi_{0}\right)+S F_{y}\right) \Omega \\
& +d_{x y}\left[D_{x} \operatorname{ctg} 2 \theta-D_{y} \operatorname{tg} 2 \theta J_{0}\left(\varphi_{0}\right)\right] \\
& +D_{y} d_{y y} J_{0}\left(\varphi_{0}\right)-D_{x} d_{x x} .
\end{aligned}
$$

Let's find wave angle $\theta$ and phase difference scanning amplitude $\varphi_{0}$ to null two last summands of (17). It means two equations should be solved for $\theta$ and $\varphi_{0}$

$$
\begin{aligned}
& D_{x} \operatorname{ctg} 2 \theta-D_{y} \operatorname{tg} 2 \theta J_{0}\left(\varphi_{0}\right)=0 \\
& D_{y} d_{y y} J_{0}\left(\varphi_{0}\right)-D_{x} d_{x x}=0 .
\end{aligned}
$$

Taking into account (12) the following solution can be obtained

$$
\begin{aligned}
J_{0}\left(\varphi_{0}\right) & =\frac{S F_{y}^{\pi / 8}}{S F_{x}^{\pi / 8}} \frac{d_{x x}}{d_{y y}} ; \\
\theta_{0} & =\frac{1}{2} \tan ^{-1} \sqrt{\frac{S F_{y}^{\pi / 8}}{S F_{x}^{\pi / 8} J_{0}\left(\varphi_{0}\right)}} .
\end{aligned}
$$

Ratio $d_{x x} / d_{y y}$ is also almost insensitive to temperature, because $X$ and $Y$ damping are almost equally changed versus temperature [4]. So, $d_{x x}$ and $d_{y y}$ can be measured at room temperature. For the gyro under test calculations give the following values: $d_{x x} / d_{y y}=0.951, J_{0}\left(\varphi_{0}\right)=0.885, \varphi_{0}=39.408 \mathrm{deg}$, $\theta_{0}=22.862 \mathrm{deg}, \omega_{\varphi}>d \Omega / d t$.

The last two summands can be nulled by using combination of LPF and band pass filter (BPF) signals extracting the second harmonic $2 \omega_{\varphi}$ frequency. After demodulation of this signals the following expressions for output signals of demodulators can be obtained
The difference of these two signals yields

$$
\begin{aligned}
z_{y}^{B P F D}-z_{x}^{B P F D} & =2 S F_{x} J_{2}\left(\varphi_{0}\right) \Omega \\
& +2 J_{2}\left(\varphi_{0}\right) D_{y} \tan 2 \theta\left(d_{y y}-d_{x y}\right) .
\end{aligned}
$$

After combination with the second equation of (16) the following equation can be obtained

$$
\begin{aligned}
z_{y}^{L P F}-z_{y}^{B P F D}-z_{x}^{B P F D}=\left[S F_{y}+2 S F_{x} J_{2}\left(\varphi_{0}\right)\right] \Omega \\
+d_{x y}\left[D_{x} \cot 2 \theta-2 D_{y} J_{2}\left(\varphi_{0}\right) \tan 2 \theta\right] \\
+D_{y} d_{y y}\left[J_{0}\left(\varphi_{0}\right)-2 J_{2}\left(\varphi_{0}\right) \tan 2 \theta\right] .
\end{aligned}
$$

As in the previous case let's find angle $\theta$ and $\varphi_{0}$ to null the two last summands of (22) which give the following two equations

$$
\begin{aligned}
D_{x} \cot 2 \theta-2 D_{y} J_{2}\left(\varphi_{0}\right) \tan 2 \theta=0 ; \\
J_{0}\left(\varphi_{0}\right)-2 J_{2}\left(\varphi_{0}\right) \tan 2 \theta=0 .
\end{aligned}
$$

So, taking into account (12) it can be obtained

$$
\begin{aligned}
\frac{J_{0}^{2}\left(\varphi_{00}\right)}{2 J_{2}\left(\varphi_{00}\right)} & =\frac{S F_{y}^{\pi / 8}}{S F_{x}^{\pi / 8}} ; \\
\theta_{00} & =\frac{1}{2} \tan ^{-1}\left[\frac{1}{J_{2}\left(\varphi_{00}\right)} \frac{S F_{y}^{\pi / 8}}{S F_{x}^{\pi / 8}}\right] .
\end{aligned}
$$

For the testing gyro the following values can be obtained: $\varphi_{00}=76.748 \mathrm{deg} \theta_{00}=39.156 \mathrm{deg}$.

\section{CONCLUSION}

Some on-line algorithms to compensate for rate CVG bias have been proposed. To implement these algorithms into practice one should be realized the possibility to modulate phase difference between oscillations along $X$ and $Y$ drive axes and to align standing wave angle $\theta$ at the prescribed value. It has experimentally been shown that the prescribed wave angle $\theta$ that is able to null bias is almost temperature insensitive, so it can be aligned by the manufacturer and will not be changed during operation. 
For more accurate applications wave angle $\theta$ should be calibrated versus temperature in order to change it during CVG operation in accordance with temperature change.

\section{REFERENCES}

[1] Chikovani, V. V; Umakhanov, E. O. and Marusyk, P. I. "The compensated differential CVG," in Proc. Gyro Technology: Symposium, 16-17 September 2008, Karlsruhe, Germany, pp. 3.1-3.8.

[2] Chikovani, V. V.; Tsiruk, H. V. "Viratory gyro accuracy parameters improving by means of excitation control." Elelectronics and Control Systems, NAU, Kyiv, no. 3 (37), 2013, pp. 43-48.

[3] Lynch, D. D. "Coriolis vibratory gyros," in Proc. Gyro Technology Simposium, 21-23 September, 1998, Stuttgart, Germany, pp. 3.1-3.14.

[4] Hopcroft, M. A.; Kim, B.; Chandorkar, S. and others "Using the temperature dependence of resonator quality factor as a termometer." Aplied physics letters, vol. 91, issue 1, July 2007.

Received 09 December

Chikovani Valeriy. Doctor of Engineering.

Member of IEEE, Education: Moscow Physical-Technical Institute, Moscow, USSR (1975).

Research interests: gyroscopy, inertial navigation system, control systems and digital information processing. Publications: 85 .

E-mail: valeriy-chikovani@rambler.ru

Tsiruk Hanna. Student.

Education: National Aviation University pre-bachelor.

Research interests: gyroscopy, control systems.

Publications: 1.

E-mail: annylee@ukr.net

\section{В. В. Чіковані, Г. В. Цірук. Компенсація зміщення нуля в диференціальному коріолісовому вібраційному} гіроскопі

Представлено різні варіанти алгоритмів компенсації зміщення нуля коріолісового вібраційного гіроскопа, які можуть застосовуватися для компенсації початкового зміщення нуля гіроскопа та його дрейфу. Також представлено експериментальні результати впливу температури на ключові параметри диференціального коріолісового вібраційного гіроскопа.

Ключові слова: диференціальний коріолісовий вібраційний гіроскоп; зміщення нуля; кут стоячої хвилі; різниця фаз.

Чіковані Валерій Валеріанович. Доктор технічних наук. Член IEEE.

Освіта: Московський фізико-технічний інститут, Москва, СРСР (1975).

Напрямок наукової діяльності: гіроскопи, інерційні навігаційні системи, системи управління та цифрова обробка інформації.

Кількість публікації: 85.

E-mail: valeriy-chikovani@rambler.ru

Цірук Ганна Вікторівна. Студентка.

Освіта: Національний Авіаційний Університет, Київ, Україна, 4 курс, (2014).

Напрямок наукової діяльності: гіроскопи, системи управління.

Кількість публікації: 1.

E-mail: annylee@ukr.net

В. В. Чиковани, А. В. Цирук. Компенсация смещения нуля в дифференциальном кориолисовом вибрационном гироскопе

Представлены различные варианты алгоритмов компенсации смещения нуля кориолисового вибрационного гироскопа, которые могут применяться для компенсации начального смещения нуля гироскопа и его дрейфа. Также, представлены экспериментальные результаты по влиянию температуры на ключевые параметры дифференциального кориолисового вибрационного гироскопа.

Ключевые слова: дифференциальный кориолисовый вибрационный гироскоп; смещение нуля; угол стоячей волны; разность фаз.

Чиковани Валерий Валерианович. Доктор технических наук. Член IEEE.

Образование: Московский физико-технический институт, Москва, СССР (1975).

Направление научной деятельности: гироскопы, инерциальные навигационные системы, системы управления и цифровая обработка информации.

Количество публикаций: 85.

E-mail: valeriy-chikovani@rambler.ru

Цирук Анна Викторовна. Студентка.

Образование: Национальный Авиационный Университет, Киев, Украина, 4 курс, (2014).

Направление научной деятельности: гироскопы, системы управления.

Количество публикаций: 1.

E-mail: annylee@ukr.net 